\title{
Autoimmune hemolytic anemia following influenza a pandemic H1N1 infection: A case report
}

\author{
Pritam Bandyopadhyay $^{*}$, Tamoghna Biswas $^{2}$, Mahuya Lahiri ${ }^{3}$ \\ ${ }^{1}$ Assistant Professor \& HOD, ${ }^{2}$ Senior Resident, ${ }^{3}$ Assistant Professor, Dept. of Pediatrics, ESIC Medical College and Hospital, \\ Kolkata, West Bengal, India
}

*Corresponding Author: Pritam Bandyopadhyay

Email: pritambanerjee86@gmail.com

\begin{abstract}
Autoimmune hemolytic anemia has been rarely reported in association with influenza virus. Here, we report a 16 month old girl with warm autoimmune hemolytic anemia following influenza A Pandemic H1N1 infection, which improved with oral corticosteroids and intravenous immunoglobulin.
\end{abstract}

Keywords: Influenza A(H1N1) infection, AHA(autoimmune hemolytic anemia), IVIg(Intravenous human gammaglobulin) administration.

\section{Introduction}

Influenza $\mathrm{A} /(\mathrm{H} 1 \mathrm{~N} 1) \mathrm{pdm} 09$ virus is a significant cause of mortality and morbidity across the globe, even in the post-pandemic period. While majority of the infected population recovers uneventfully, the severity is more pronounced in vulnerable age groups like children who are prone to suffer from complications of the disease. Here, we present a case of influenza $\mathrm{A} /(\mathrm{H} 1 \mathrm{~N} 1) \mathrm{pdm} 09$ with autoimmune hemolytic anemia, a rarely reported association.

\section{Case Report}

A 16 month old girl from South 24 Pargana district of West Bengal, India was admitted in our pediatric intensive care unit with a history of cough and coryza for five days followed by high grade fever and respiratory distress with inability to suck well for one day. On admission, the child was lethargic, and tachypneic with marked intercostal and subcostal retractions with oxygen saturation below $90 \%$ in room air. Auscultation revealed bilateral wheeze and crackles with decreased air entry on the right side. Initial chest $X$ ray (Fig. 1) was suggestive of right sided pneumonia. With the provisional diagnosis of right upper lobar pneumonia child was started on empirical antibiotics with intravenous amoxicillinclavulunate which was subsequently upgraded to intravenous meropenem and vancomycin due to the deteriorating respiratory status. Supportive treatment was continued which included supplemental oxygen, intravenous fluids and nebulization with salbutamol and ipratropium. Blood and urine cultures were negative. Nucleic Acid Amplification Test done on throat swab specimen at the regional virus diagnostic laboratory, was positive for Influenza A Pandemic H1N1 infection. The child was started on a course of antiviral oral oseltamivir $30 \mathrm{mg}$ BD for five days. The child showed dramatic improvement within 48 hours of starting oseltamivir with complete subsidence of respiratory symptoms within five days. However, even after 10 days of admission, a low grade fever persisted and the child was developing clinically significant pallor, which was absent at the time of admission. Complete hemogram showed anemia (hemoglobin 5.1 $\mathrm{g} / \mathrm{dL}$, red cell count $\left.1.1 \mathrm{X} 10^{6} / \mu \mathrm{L}\right)$ with leucocytosis (total leucocyte count $22700 / \mu \mathrm{L}$ with $59 \%$ neutrophils, $32 \%$ lymphocytes, $6 \%$ monocytes and $3 \%$ eosinophils). Reticulocytosis (reticulocyte count $3.5 \%)$ and elevated lactate dehydrogenase (1340 U/L) suggested an acquired hemolytic anemia. An immune mediated process was suspected as there was problem in cross matching for blood group determination as reported in our blood bank. Polyspecific direct antiglobulin test (DAT) or direct Coombs test was positive, and monospecific Coombs test showed IgG positivity at 37 degree celsius typical of warm autoimmune hemolytic anemia. Intravenous immunogloubulin (IVIg) was administered and oral prednisolone was started at a dose of $2 \mathrm{mg} / \mathrm{kg} /$ day. An increase in hemoglobin was apparent within five days after start of steroids, and the child was discharged in a clinically stable condition with tapering course of oral corticosteroids. On follow up after two weeks, DAT was negative and hemoglobin level was normal without blood transfusion. 


\section{Discussion}

AIHA is a relatively uncommon disease in children. While accurate estimates of incidence are lacking, a French observational study ${ }^{1}$ reported a rate of 0.2 cases/1,000,000 under-20 population. AIHA is characterized by premature lysis of red blood corpuscles mediated by autoantibodies, depending on the thermal range of which the disease is classified into warm antibody, cold antibody and mixed type AIHA. Secondary AIHAs have been reported to be associated with infections, drug exposure, autoimmune disorders, lymphoproliferative disorders, malignancies etc. Among infections causing secondary AIHA, Mycoplasma pneumonia, Epstein-Barr virus, cytomegalovirus, parvovirus, hepatitis $\mathrm{A}$ and $\mathrm{E}$ virus, etc are significant. However, influenza virus has been rarely reported to be associated with $\mathrm{AIHA}^{2}$. Till date, only a few case reports ${ }^{3-6}$ exist in the global scientific literature about a possible association between influenza virus infection and secondary AIHA. In 2009, Schoindre et $\mathrm{al}^{3}$ reported a case of cold agglutinin syndrome (CAS) in a 60 years old woman with diabetic nephropathy following influenza A H1N1 infection. Cold agglutinin disease following influenza $\mathrm{A}$ infection has also been reported by Ahn et $\mathrm{al}^{4}$ in a 58 years old female from Brazil. Shizuma reported a case of AIHA following influenza $\mathrm{A}$ in a patient with alcoholic liver disease, who responded initially to prednisolone, but later succumbed due to hepatorenal syndrome. Recently in 2017, a pediatric center from North India has also reported ${ }^{6}$ a case of AIHA in a one year old girl with influenza A H1N1 infection, who responded favorably to treatment with oseltamivir and intravenous immunoglobulin. Our ${ }^{1}$ patient was treated with both oral steroids and IVIG, in view of the fulminant clinical course and considering the increased likelihood of severe complications in this vulnerable age group. Although our patient received Oseltamivir as antiviral agent against Influenza $\mathrm{A}(\mathrm{H} 1 \mathrm{~N} 1)$ infection, but the medicine is not a known agent to cause drug induced $\mathrm{AHA},{ }^{8}$ except in one report, ${ }^{9}$ but there also AHA was recovered 2 weeks after withdrawal of antiviral agent and concomitant steroid therapy whereas in drug induced cases respond is immediate following omission of the drug. So here AHA is more likely to be caused by $\mathrm{H} 1 \mathrm{~N} 1$ infection itself, rather oseltamivir induced as in our case. A case reported by Chen $\mathrm{H} 1$ et $\mathrm{al}^{7}$ that AHA with thrombocytopenia treated with Methylprednisolone had succumbed due to multiorgan failure.

\section{Conclusion}

This case presentation suggested that though rare, autoimmune hemolytic anemia can be a potentially devastating complication of influenza A infection. Keeping a low threshold of suspicion is imperative for early recognition and prompt treatment of this condition.

\section{Source of funding}

None.

\section{Conflict of interest}

None.

\section{References}

1. Aladjidi N, Leverger G, Leblanc T, Picat MQ, Michel G, Bertrand $\mathrm{Y}$ et al. Centre de Référence National des Cytopénies Auto-immunes de l'Enfant (CEREVANCE). New insights into childhood autoimmune hemolytic anemia: a French national observational study of 265 children. Haematologica 2011;96(5):655-63. DOI: 10.3324/hematol.2010.036053.

2. Shizuma T. Autoimmune hemolytic anemia following influenza virus infection or administration of influenza vaccine. J Blood Disorders Transf 2014;5(3):1000200. DOI: $10.4172 / 2155-9864.1000200$

3. Schoindre Y, Bollée G, Dumont MD, Lesavre P, Servais A. Cold agglutinin syndrome associated with a 2009 influenza A H1N1 infection. Am J Med 2011;124(2):e1-2.DOI: https://doi.org/10.1016/j.amjmed.2010.05.015

4. Ahn M, Rehr MJ, Santarelli GE. Cold hemolytic anemia: a rare complication of influenza A. The Journal of Community and Supportive Oncology. 2017;15(6):e335e338. DOI :https://doi.org/10.12788/jcso.0346

5. Shizuma T. [A case of autoimmune hemolytic anemia caused by type A influenza infection in a patient with alcoholic liver cirrhosis]. Kansenshogaku Zasshi 2010;84(3):296-9. Japanese. DOI:

10.11150/kansenshogakuzasshi.84.296

6. Singh A, Mandal A, Patel A, Mishra S. Autoimmune Haemolytic Anaemia-A Spectrum of Presentation in Children. J Clin Diagn Res 2017;11(9):SR01-SR02. DOI: 10.7860/JCDR/2017/27895.10646

7. Chen H1, Jia XL, Gao HM, Qian SY. Comorbid presentation of severe novel influenza $(\mathrm{H} 1 \mathrm{~N} 1)$ and Evans syndrome: a case report. Chin Med J (Clin Res Ed) 2011;282:1743-6. DOI: 10.3760/cma.j.issn.036669999.2011.11.026

8. Garratty G. Immune hemolytic anemia associated with drug therapy. Blood Rev 2010;24:143-50. DOI: https://doi.org/10.1016/j.blre.2010.06.004

9. Yagi Y, Yagi Y, Sageshima H, Miyata Y. A case of infant with hemolytic anemia after oral oseltamivir administration. Acta Paediatr Jpn 2004;108:649-51. https://ci.nii.ac.ip/naid/10014204484\#cinii

How to cite: Bandyopadhyay P, Biswas T, Lahiri M. Autoimmune hemolytic anemia following influenza a pandemic H1N1 infection: A case report. IP J Paediatr Nurs Sci 2020;3(1):28-9. 